\title{
CARACTERIZAÇÃO DE BIOMASSAS PARA A BRIQUETAGEM
}

\author{
Diego Aleixo da Silva ${ }^{1 *}$, Fabio Minoru Yamaji² ${ }^{2}$ João Lúcio de Barros ${ }^{3}$, Alessandra Luzia da Róz ${ }^{4}$, \\ Gabriela Tamy Nakashima ${ }^{5}$ \\ ${ }^{1 *}$ Universidade Federal de São Carlos, Curso de Engenharia Florestal, Sorocaba, São Paulo, Brasil - \\ diegoaleixo.florestal@gmail.com \\ ${ }^{2}$ Universidade Federal de São Carlos, Departamento de Ciências Ambientais, Sorocaba, São Paulo, Brasil - fmyamaji@ufscar.br \\ ${ }^{3}$ Instituto Federal de Educação, Ciência e Tecnologia de São Paulo, Itapetininga, São Paulo, Brasil - barros.jlucio@ gmail.com \\ ${ }^{4}$ Universidade Federal de São Carlos, Sorocaba, São Paulo, Brasil - alessandra.roz@ gmail.com \\ ${ }^{5}$ Universidade Federal de São Carlos, Programa de Pós-Graduação em Planejamento e Uso de Recursos Renováveis, Sorocaba, São \\ Paulo, Brasil - gabi.tami@gmail.com
}

Recebido para publicação: 30/01/2015 - Aceito para publicação: 09/09/2015

\begin{abstract}
Resumo
O objetivo deste trabalho foi caracterizar a produção de briquetes feita a partir de quatro diferentes biomassas residuais. Foram utilizados os resíduos de serragem de Eucalyptus sp, serragem de Pinus sp, bagaço de cana-de-açúcar (Saccharum officinarum L.) e palha de cana-de-açúcar. Os resíduos foram tratados para que obtivessem $12 \%$ de umidade e uma granulometria inferior a 1,70 $\mathrm{mm}$. Foram produzidos 15 briquetes para cada um dos quatro tratamentos. A pressão utilizada foi de $1250 \mathrm{kgf.cm}$ 2 durante 30 segundos. Os briquetes obtiveram densidades que oscilaram 0,88 a $1,11 \mathrm{~g} . \mathrm{cm}^{-3}$. Isto representou uma faixa de 5 a 14 vezes a menos de ocupação de volume para uma mesma quantidade de massa. O poder calorifico foi de $19.180 \mathrm{~J} . \mathrm{kg}^{-1}$ e $20.315 \mathrm{~J} . \mathrm{kg}^{-1}$ para as serragens de eucalipto e pinus respectivamente. Para o bagaço e palha de cana os valores foram de $18.541 \mathrm{~J} . \mathrm{kg}^{-1}$ e $15.628 \mathrm{~J}_{\mathrm{kg}}{ }^{-1}$. A palha da cana-de-açúcar apresentou um teor de cinzas de $12 \%$. As expansões dos tratamentos oscilaram 4 a $9 \%$ e as resistências mecânicas variaram de 1,215 MPa à 0,270 MPa. Todos os briquetes se mostraram resistentes para um empilhamento superior a $10 \mathrm{~m}$ de altura. $\mathrm{O}$ procedimento adotado pode ajudar a diminuir o espaço de estocagem e de transporte.

Palavras-chave: Resíduo; biocombustível; energia; compressão; empilhamento.
\end{abstract}

\begin{abstract}
Characterization of biomass for briquetting. This research aims to characterize the production of briquettes from four different biomasses. We used residues such as Eucalyptus sp sawdust, Pinus sp sawdust, sugarcane bagasse (Saccharum officinarum L.) and sugarcane straw. The residues were treated to obtain $12 \%$ moisture content and particle size less than $1.70 \mathrm{~mm}$. We produced 15 briquettes for each treatment. The pressure used was $1250 \mathrm{kgf.cm}{ }^{-2}$ for 30 seconds. The briquettes obtained densities ranged from 0.88 to $1.11 \mathrm{~g} . \mathrm{cm}^{-3}$. This represented a range of 5 to 14 times less volume occupancy for the same amount of mass. The high heating value (HHV) was $19,180 \mathrm{~J}^{\mathrm{kg}}{ }^{-1}$ and $20,315{\mathrm{~J} . \mathrm{kg}^{-1}}^{-}$for eucalyptus and pine sawdust respectively. The HHV for the bagasse was $18,541 \mathrm{~J} . \mathrm{kg}^{-1}$ and for straw was $15,628 \mathrm{~J} . \mathrm{kg}^{-1}$. The straw presented an ash content of $12 \%$. The expansions of the treatments ranged 4 to $9 \%$ and mechanical resistances ranging from 1,215 MPa to $0,270 \mathrm{MPa}$. All briquettes were resistant to a higher stacking to $10 \mathrm{~m}$ high. The methods can help to decrease the space of storage and transport.

Keywords: Waste; biofuel; energy; compression; stacking.
\end{abstract}

\section{INTRODUÇÃO}

A matriz energética mundial energia é sustentada a partir de fontes fósseis, o que gera emissões de poluentes locais, gases de efeito estufa e põem em risco o suprimento que ainda resta no planeta. É preciso mudar esses padrões estimulando as energias renováveis, e, nesse sentido, o Brasil apresenta uma condição bastante favorável em relação ao resto do mundo (GOLDEMBERG; LUCON, 2007). Pois segundo a Empresa de Pesquisa Energética (EPE) (2014), em 2013, 41,0\% do consumo de energia no Brasil era proveniente de fontes renováveis, ao passo que no mundo eram apenas $13,0 \%$.

FLORESTA, Curitiba, PR, v. 45, n. 4, p. 713 - 722 , out. / dez. 2015.

Silva, D. A. da. et al.

ISSN eletrônico 1982-4688 / ISSN impresso 0015-3826 
Em 2013 foram geradas 609,9 TWh de energia elétrica no Brasil, dos quais 70,6\% foram provenientes da matriz hidráulica. Entretanto devido às condições hidrológicas houve uma diminuição em $8,45 \%$ da energia hidrelétrica em relação a 2012. Isso refletiu em um aumento de energia térmica, acarretando em aumento de 5,2\% da matriz não renovável (EPE, 2014).

Diversificar a matriz energética para a eletricidade é uma preocupação tendo em vista as eventualidades climáticas cada vez mais instáveis. Principalmente quando influem diretamente nas usinas hidrelétricas brasileiras. Um bom resultado é que o uso das biomassas destinadas à geração de eletricidade tem aumentado, mas ainda é um valor inferior a 10\% (EPE, 2014).

Assim, vem ocorrendo uma grande valorização da produção biomassa como fonte de energia. Esta se desenvolve de forma alternativa para minimizar a geração de resíduos e impactos ambientais. Além disso, o país é um paradigma mundial pelo seu vigoroso programa de biomassa moderna no setor de transportes baseado no etanol (GOLDEMBERG; LUCON, 2007).

Como o país possui proporções continentais, sendo o quinto maior país do mundo, nele tem grande destaque o setor florestal e ao setor sucro-alcoleira. De acordo com o IBA (2015) os principais gêneros florestais plantados no Brasil, Eucalyptus sp e Pinus sp, apresentam uma área com 5,56 milhões de ha e 1,59 milhões ha, respectivamente. Já no setor sucroalcooleiro, a área para a produção brasileira de cana de açúcar foi 9,0 milhões de ha na safra 2014/15 (CONAB, 2015).

No estado de São Paulo a lei $n^{\circ} 11.241 / 2002$ veta a queima de cana-de-açúcar pré-colheita, isto devido aos impactos negativos na saúde coletiva e no meio ambiente. Assim com resultado desta lei, um novo resíduo do processo não será apenas o bagaço da cana, mas também a palha, já que a mesma era eliminada no processo de queima. Isto mostra uma possibilidade de maior oferta de biomassa no setor sucroalcooleiro que pode ser direcionado para a matriz energética brasileira.

Ainda no setor florestal, o eucalipto e o pinus servem de matéria prima para alimentar diversos mercados como o de celulose, madeira serrada, compensados, produtos de maior valor agregado (PMVA), móveis, ferro gusa e carvão vegetal. Em 2014 o setor florestal foi responsável por gerar mais quatro milhões de empregos e representar 1,1\% do produto interno bruto do Brasil (IBA, 2015).

Durante o processo de transformação da madeira de eucalipto e pinus são geradas toneladas de resíduos. Uma alternativa sustentável para o aproveitamento desses resíduos seria a compactação a fim de se obter um material altamente energético para a geração de energia térmica, o qual ocuparia um menor volume.

Alguns dos principais tipos de resíduos lignocelulósicos utilizados na briquetagem são a palha de milho, pó de serra, serragem de madeira (eucalipto, pinus, seringueira entre outros), e resíduos de compensado (principalmente de pinus), casca de arroz e bagaço de cana (QUIRINO, 2014).

Nessa nova linha de pesquisa, tem grande destaque a produção de briquetes, os quais podem ser feitos através da compactação de resíduos lignocelulósicos, uma alternativa empregada dentro do meio florestal e rural visando à eliminação de resíduos com potencial de queima (FLORES et al., 2009). Como o aproveitamento é a partir de uma determinada biomassa, um parâmetro que deve ser considerado é o teor de umidade, o que de acordo com Quirino (1991) e Lucena et al. (2008) envolve um teor de umidade de 10 a $12 \%$ no processo de compactação. Entretanto, a umidade da biomassa interfere na produção de calor por unidade de massa, assim conforme menor for o teor de umidade, maior será o poder calorífico do material (VALE et al., 2000).

Ainda assim, o processo de concentração de energia é muito eficiente, pois de acordo com Quirino (1991) a briquetagem pode resultar em valor de 5 vezes mais de energia do que na forma não compactada. O resultado ocorre pela diminuição do espaço de volume inicial e pelo preenchimento de mais massa para se alcançar o mesmo volume inicial, assim consecutivamente o teor de carbono disponível para a queima.

Outra variável de importante análise para a confecção de briquetes é a granulometria do material, que pode interferir na sua compactação, resultando em valores diferentes da força máxima suportada pelo material. Costa et al. (2010) encontraram valores diferentes na força máxima de briquetes com granulometrias diferentes, todos com material de origem oriundo de Pinus sp.

O objetivo do presente trabalho foi caracterizar quatro diferentes tipos de biomassa para a briquetagem. Os resíduos utilizados foram a serragem de Eucalyptus sp., a serragem de Pinus sp., bagaço de cana-de-açúcar e palha de cana-de-açúcar. O grande espaço e volume de ocupação foram características indesejáveis dos materiais em seus locais de origem, chegando a formarem leiras de resíduos. 


\section{MATERIAL E MÉTODOS}

Este trabalho foi desenvolvido no Laboratório de Bioenergia e Materiais Lignocelulósicos, da Universidade Federal de São Carlos - campus Sorocaba, SP. Foram utilizados os resíduos de serragem de Eucalyptus sp, serragem de Pinus sp, bagaço de cana-de-açúcar (Saccharum officinarum L.) e palha de cana-de-açúcar.

A aquisição das serragens foi em uma serraria de pequeno porte (processamento menor que $10.000 \mathrm{~m}^{2} / \mathrm{ano}$ ) no distrito de Itapetininga, SP. O bagaço de cana foi adquirido na Usina Santa Rosa, Porto Feliz, SP, a qual produz etanol e açúcar. A palha de cana foi adquirida na Fazenda Corredeira, São Carlos, SP, a qual planta cana-de-açúcar.

\section{Preparação dos briquetes}

Após a coleta dos materiais, os mesmos foram secos em estufa a $105{ }^{\circ} \mathrm{C}\left( \pm 2{ }^{\circ} \mathrm{C}\right)$ por $24 \mathrm{~h}$ a fim de reduzir o seu alto teor de umidade inicial e, em seguida, foram armazenados em sacos de polietileno a temperatura ambiente $\left(25^{\circ} \mathrm{C} \pm 2{ }^{\circ} \mathrm{C}\right)$ até sua utilização.

A moagem das biomassas foi realizada separadamente em um moinho de facas tipo Willey, Marconi - MA 340, utilizando velocidade fixa de $1750 \mathrm{rpm}$ e peneira de abertura de $1,70 \mathrm{~mm}$. Assim a faixa granulométrica utilizada foi a de partículas menores que $1,70 \mathrm{~mm}$. Na sequência os materiais foram levados novamente à estufa a $105^{\circ} \mathrm{C}\left( \pm 2{ }^{\circ} \mathrm{C}\right)$ até atingirem peso constante. Após a secagem, foi possível a padronizar a umidade das matérias-primas com teor de umidade de $12 \%$. $\mathrm{O}$ ajuste do teor de umidade foi feito com auxílio de um borrifador e balança semianalítica. O teor de umidade de $12 \%$ foi escolhido por estar dentro da faixa considerada ideal para a fabricação de briquetes (QUIRINO, 1991; LUCENA et al, 2008; KONISHI et al, 2011). Foram realizados quatro tratamentos para a briquetagem: tratamento T1 (serragem de eucalipto), tratamento T2 (serragem de pinus), tratamento T3 (bagaço de cana-de-açúcar) e tratamento T4 (palha de cana-de-açúcar).

Foram produzidos quinze briquetes para cada um dos tratamentos. Todos os briquetes foram preparados empregando-se massa de $20 \mathrm{~g}$, pressão de $1250 \mathrm{kgf.cm}{ }^{-2}$ e tempo de prensagem de $30 \mathrm{~s}$. Na prensagem não foram utilizados agentes aglutinantes e nem aquecimento. O processo de compactação do material em forma de briquete foi realizado em uma prensa hidráulica (Marconi - MA 098/CP) com o auxílio de moldes cilíndricos de aço inox com diâmetro de $3,5 \mathrm{~cm}$ e altura de $16 \mathrm{~cm}$.

\section{Determinação da granulometria}

Para a determinação granulométrica utilizou-se o agitador orbital de peneiras com batidas intermitentes marca Marconi, modelo MA 750. Foram utilizadas $100 \mathrm{~g}$ da biomassa e tempo de agitação de três minutos. A classificação granulométrica do material foi determinada utilizando-se as peneiras com abertura de 1,$70 ; 0,85 ; 0,43 ; 0,25 ; 0,15 \mathrm{~mm}$ e o fundo. As biomassas foram analisas antes e após a moagem.

\section{Densidade}

Para a determinação da densidade a granel das biomassas, foram utilizados um béquer de $1 \mathrm{~L}$ de capacidade e de peso conhecido. A determinação ocorreu por uma média das triplicatas, seguindo uma metodologia adaptada da norma ABNT NBR 6922/81. Após o processo de briquetagem foram determinadas as densidades aparentes das amostras de cada tratamento.

Para a densidade aparente foram utilizadas 15 amostras por tratamento. A densidade dos resíduos foi determinada nas condições antes da briquetagem (densidade a granel) e após briquetagem (densidade aparente dos briquetes), ambos a $12 \%$ de umidade.

\section{Análise imediata}

Todas as análises se deram na forma de triplicatas. Os teores de cinzas foram determinados conforme a ASTM D1102-84 (2007), e os teores de voláteis foram obtidos em triplicata conforme a norma ABNT NBR 8112/86. O carbono fixo foi calculado subtraindo-se de $100 \%$ a soma dos teores de voláteis e de cinzas. O poder calorífico superior (PCS) foi obtido com auxílio de uma bomba calorimétrica IKA C200, com base na norma ABNT NBR 8633/84. 


\section{Características físico-mecânicas}

A expansão longitudinal dos tratamentos foi calculada pela mensuração da altura dos corpos-deprova no mesmo sentido de aplicação da força de prensagem. Foram realizadas as mensurações dos corpos-de-prova após a briquetagem e antes do ensaio de compressão diametral, ou seja, nove dias após a briquetagem.

No intervalo de $24 \mathrm{~h}$ foram coletadas as alturas de cada tratamento, assim foi possível calcular a estabilidade dimensional dos briquetes. tratamento.

No ensaio de expansão e de estabilidade dimensional foram utilizadas 15 amostras por

Após acondicionamento das amostras em sacos de polietileno lacrados em temperatura média de $25^{\circ} \mathrm{C}$ por 9 dias. A análise de compressão foi realizada empregando-se uma máquina universal de ensaios EMIC - DL30000, com célula de carga de $500 \mathrm{kgf}$, a uma velocidade constante de $3 \mathrm{~mm} / \mathrm{min}$. O ensaio foi realizado a partir de uma adaptação da norma ABNT (1994) para determinação da resistência a tração por compressão diametral em amostras cilíndricas de concreto e argamassa.

O número de amostras empilhadas verticalmente $\left(\mathrm{N}_{\mathrm{emp}}\right)$ foi calculado pela força máxima de ruptura $\left(\mathrm{F}_{\text {Mrup }}\right)$, por um fator de segurança $\left(\mathrm{f}_{\mathrm{s}}\right)$ e pela massa média das amostras $(20 \mathrm{~g})$. $\mathrm{O} \mathrm{N} \mathrm{N}_{\mathrm{emp}}$ representa a quantidade máxima de amostras empilhadas que um tratamento suporta. $\mathrm{O} \mathrm{F}_{\text {Mrup }}$ foi fornecido pelo ensaio de compressão. $\mathrm{O} \mathrm{f}_{\mathrm{s}}$ foi considerado como $300 \%$, ou seja, diminui o $\mathrm{F}_{\text {Mrup }}$ em três vezes. A equação 1 resume o cálculo do $\mathrm{N}_{\mathrm{emp}}$.

$$
N_{\text {emp }}=\frac{F_{\text {Mrup }}}{f_{S} \times 0,020}
$$

A partir do $\mathrm{N}_{\mathrm{emp}}$ é possível calcular a altura do empilhamento $\left(\mathrm{H}_{\mathrm{emp}}\right)$. Como o diâmetro das amostras é em media $3,5 \mathrm{~cm}$ a $\mathrm{H}_{\mathrm{emp}}$ é dada pela equação 2.

$$
H_{e m p}=\frac{N_{e m p} \times 3,5}{100}
$$

A porcentagem de friabilidade foi realizada por meio do ensaio de resistência ao impacto e abrasão, seguindo uma adaptação da norma ABNT NBR 8740/85, recomendada por Barros (2014), com auxílio de um friabilômetro. Foi utilizado uma rotação de $30 \mathrm{rpm}$, em um ensaio de 15 minutos. Foram utilizadas quatro amostras para cada ensaio. Ao fim do ensaio, separou-se com uma peneira de abertura de $4 \mathrm{~mm}$ a massa da biomassa ainda aglutinada e compactada. O material restante reflete a friabilidade dos tratamentos.

\section{Análise estatística}

O delineamento experimental foi inteiramente casualizado, constituindo quatro tratamentos. Respeitando-se as exigências estatísticas os dados coletados foram submetidos ao Teste de Shapiro-Wilk, à análise de variância (ANOVA) e ao teste Tukey (com 5\% de significância). Todos os testes foram calculados com o software Tinn- $\mathrm{R}^{\circledR}$, versão 2.14.2.

\section{RESULTADOS E DISCUSSÃO}

\section{Classificação granulométrica}

Na figura 1 e na figura 2 é possível relacionar as diferenças na distribuição granulométrica dos materiais para o um melhor procedimento da briquetagem.

Após a moagem da serragem de eucalipto, é possível dizer que houve um aumento das partículas com tamanho menor que $0,50 \mathrm{~mm}$, aproximadamente, e um decréscimo das partículas com tamanho maior que $0,50 \mathrm{~mm}$. Já para a serragem de pinus o aumento ocorre antes de $0,25 \mathrm{~mm}$ aproximadamente.

$\mathrm{Na}$ moagem do bagaço de cana houve um aumento das partículas com tamanho menor que $0,75 \mathrm{~mm}$, aproximadamente, e um decréscimo das partículas com tamanho maior que $0,75 \mathrm{~mm}$. Já para a palha de cana ocorre um aumento, para partículas menores que $0,50 \mathrm{~mm}$ aproximadamente.

A granulometria é um parâmetro de extrema importância no processo de briquetagem que influência na resistência do produto final. Pois a presença de partículas menores auxilia a preencher espaços vazios (FERRAGUTI et al., 2012; GONÇALVES et al., 2013; NAKASHIMA, 2013). 

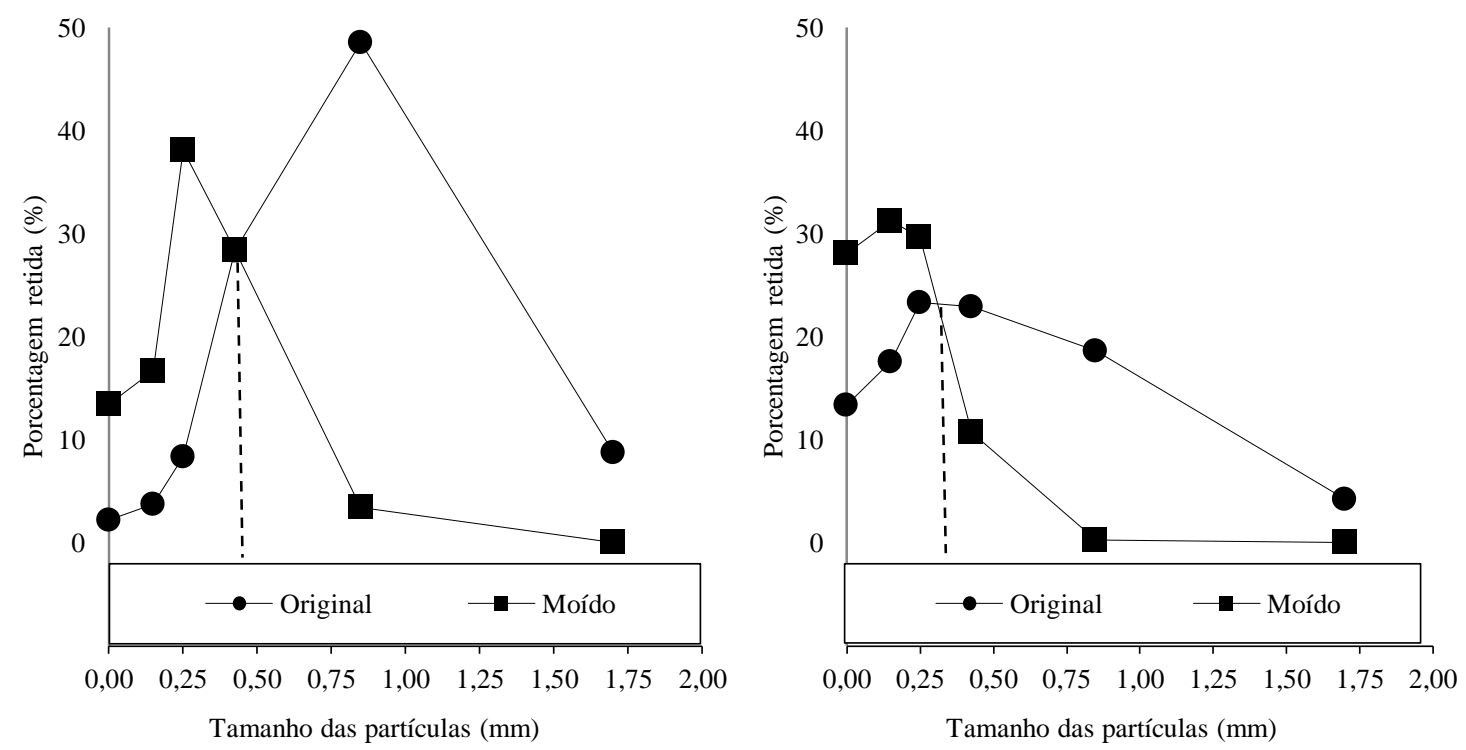

Figura 1. Análise granulométrica da serragem do eucalipto (à esquerda) e da serragem de pinus (à direita).

Figure 1. Particle size analysis of sawing eucalyptus (left) and pine sawdust (right).
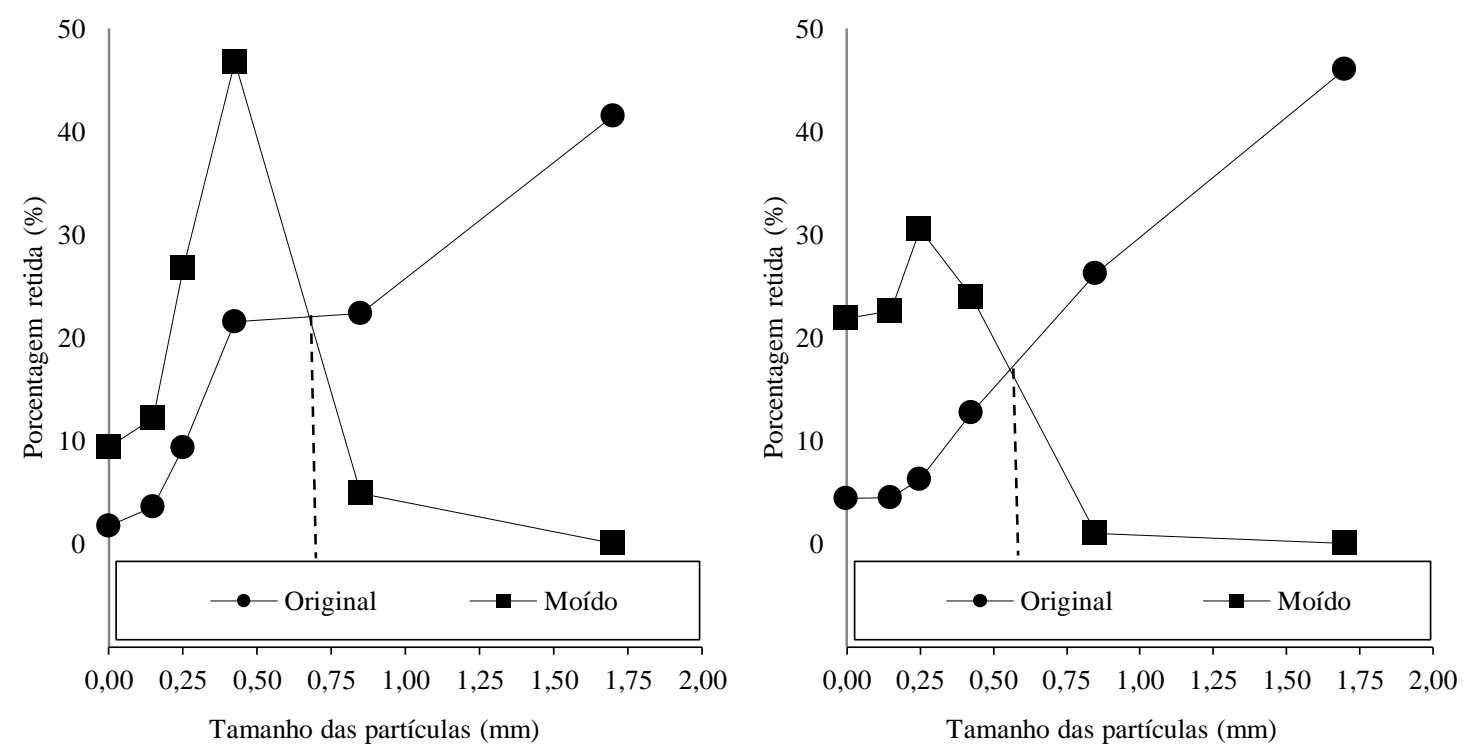

Figura 2. Análise granulométrica do bagaço de cana (à esquerda) e da palha de cana (à direita).

Figure 2. Particle size analysis of sugarcane bagasse (left) and sugarcane straw (right).

\section{Densidade}

A partir da tabela 1 é possível ver que ocorreu um aumento da densidade nos quatro tratamentos. Para as biomassas briquetadas, verificou-se uma redução de volume de 4,6 à 14,3 vezes. $\mathrm{O}$ aumento na densidade dos briquetes representa uma diminuição do volume das biomassas (uma maior de concentração de massa em um mesmo espaço). Isto demonstra a eficiência dos briquetes em termos de transporte e estocagem.

Yamaji et al. (2013) e Chrisostomo (2011) encontraram densidades menores para os briquetes de serragem de eucalipto (T1) e de pinus (T2). Os valores da literatura variaram de 0,80 à $0,87{\mathrm{~g} . \mathrm{cm}^{-3}}$ para a

FLORESTA, Curitiba, PR, v. 45, n. 4, p. 713 - 722 , out. / dez. 2015.

Silva, D. A. da. et al.

ISSN eletrônico 1982-4688 / ISSN impresso 0015-3826 
serragem de eucalipto e oscilaram de 0,85 à $0,86 \mathrm{~g} . \mathrm{cm}^{-3}$ para a serragem de pinus. Yamaji et al. (2013) encontraram um valor de $0,66 \mathrm{~g} . \mathrm{cm}^{-3}$ para briquetes de bagaço de cana-de-açúcar enquanto Chrisostomo (2011) encontrou um valor próximo ao verificado nesse trabalho $\left(0,84 \mathrm{~g} \cdot \mathrm{cm}^{-3}\right)$. Brasil et al. (2015) encontraram uma densidade de $0,9 \mathrm{~g} . \mathrm{cm}^{-3}$ para briquetes de bagaço de cana-de-açúcar usando uma granulometria menor que $0,4 \mathrm{~mm}$.

Tabela 1. Densidade das amostras, com $12 \%$ a umidade na base seca, na forma natural e compactada. Taxa de aumento relativo (TAR).

Table 1. Density of samples up to $12 \%$ moisture on dry basis, natural and compressed form. Increased relative rate (TAR).

\begin{tabular}{lcccc}
\hline \multirow{2}{*}{ Forma } & \multicolumn{4}{c}{ Densidade com 12\% de umidade $\left(\mathbf{g . c m}^{-3}\right)$} \\
\cline { 2 - 5 } & T1 & T2 & T3 & T4 \\
\hline Natural & $0,12\left( \pm 1,0 \times 10^{-3}\right)$ & $0,18\left( \pm 1,8 \times 10^{-3}\right)$ & $0,06\left( \pm 2,8 \times 10^{-3}\right)$ & $0,07\left( \pm 3,0 \times 10^{-3}\right)$ \\
Briquete & $0,92\left( \pm 0,8 \times 10^{-3}\right)$ & $1,00\left( \pm 0,6 \times 10^{-3}\right)$ & $0,88\left( \pm 0,4 \times 10^{-3}\right)$ & $1,11\left( \pm 1,4 \times 10^{-3}\right)$ \\
\hline TAR $(\%)$ & 685 & 462 & 1267 & 1431 \\
\hline
\end{tabular}

Os briquetes de Yamaji et al. (2013) foram feitos com uma granulometria de 0,85 à $0,15 \mathrm{~mm}$ e teor de umidade de $12 \%$. Já Chrisostomo (2011) usou uma faixa granulométrica de 0,85 à $0,43 \mathrm{~mm}$ e um teor de umidade de $10 \%$. Assim, as diferenças de densidade dos tratamentos podem ser explicadas pela maior amplitude granulométrica da metodologia usada. Gonçalves et al. (2013) obtiveram maior densidade para briquetes de eucalipto no tratamento que abrangeu uma maior de faixa tamanho de partículas $(1,70$ à $0,15 \mathrm{~mm})$ em relação aos de tratamentos de frações maior tamanho (menor área de contato).

\section{Análise imediata}

A tabela 2 mostra os valores médios de características químicas e energéticas das biomassas. O maior poder calorífico superior (PCS) foi apresentado pela serragem de pinus (20.315 J.kg-1). Já o menor poder calorífico (15.627 J.kg ${ }^{-1}$ ) foi constado para o tratamento T4 (palha de cana-de-açúcar) que foi a biomassa com maior quantidade de teor de cinzas (12,19\%). Chrisostomo (2011) encontrou valores de poder calorifico superior de $19.573{\mathrm{~J} . \mathrm{kg}^{-1}}$ para a serragem pinus, de $18.573{\mathrm{~J} . \mathrm{kg}^{-1}}^{-1}$ para a serragem de eucalipto e de $17.325 \mathrm{~J}_{\mathrm{kg}}{ }^{-1}$ para o bagaço de cana-de-açúcar.

Tabela 2. Análise química e poder calorifico superior (PCS) das biomassas analisadas para a briquetagem.

Table 2. Chemical analysis and higher calorific power (PCS) of analyzed biomass for briquetting.

\begin{tabular}{lccccccc}
\hline Tratamento & T.C $(\boldsymbol{\%})$ & $\boldsymbol{\sigma}( \pm)$ & T.Vol. $(\boldsymbol{\%})$ & $\boldsymbol{\sigma}( \pm)$ & C.F $(\boldsymbol{\%})$ & $\boldsymbol{\sigma}( \pm)$ & PCS $\left(\mathbf{J . k g}^{-\mathbf{1}}\right)$ \\
\hline T1 & 1,57 & 0,22 & 80,54 & 0,14 & 17,90 & 0,30 & 19.179 \\
T2 & 0,23 & 0,15 & 81,16 & 0,49 & 18,61 & 0,35 & 20.315 \\
T3 & 1,42 & 0,11 & 80,69 & 0,85 & 17,89 & 0,74 & 18.540 \\
T4 & 12,19 & 1,04 & 70,59 & 1,36 & 17,22 & 2,40 & 15.627 \\
\hline
\end{tabular}

Teor de cinzas (T.C). Teor de voláteis (T.Vol.). Carbono fixo (C.F). Desvio padrão( $\sigma)$.

\section{Resistência mecânica}

Todas as características mecânicas avaliadas na produção de briquetes apresentaram repostas distintas entre si, com um nível significância de $99,9 \%$ de confiança, conforme é mostrado na tabela 3 . No teste de Shapiro-Wilk também se confirmou uma distribuição normal de todas as amostras coletadas.

Para a característica de expansão longitudinal verificou-se que os valores médios dos tratamentos variaram entre 3,85 e 9,00\%. Esses valores podem ser considerados pequenos se comparados aos encontrados por Yamaji et al. (2013), onde a expansão longitudinal foi de 18\% para briquetes de bagaço de cana-de-açúcar e de 35\% para briquetes de capim. Brasil et al. (2015) encontraram uma expansão volumétrica de $22 \%$ para briquetes de cana-de-açúcar. O tratamento T1 (serragem de eucalipto) apresentou uma expansão longitudinal de $9,00 \%$. Ainda que tenha apresentado a maior expansão entre os quatro tratamentos, foi o tratamento que obteve a maior resistência mecânica (1,215 MPa). Isso demonstra que essa expansão longitudinal pode ser considerada normal para a serragem de eucalipto, pois 
não afetou a qualidade do briquete. $\mathrm{O}$ tratamento T3 (bagaço de cana-de-açúcar) apresentou uma expansão longitudinal de $8,10 \%$. Entretanto, foi o tratamento que apresentou a menor resistência mecânica $(0,27 \mathrm{MPa})$. Os resultados mostram que cada material apresenta um comportamento diferente na expansão longitudinal. Para analisar os ensaios de expansão longitudinal é preciso considerar o tipo de biomassa utilizado. A análise isolada pode levar a interpretações equivocadas.

O tratamento T4 (palha de cana-de-açúcar) foi estatisticamente superior em relação à característica densidade, apresentando média de $1,11 \mathrm{~g} . \mathrm{cm}^{-3}$. Os menores valores médios de densidade foram evidenciados para os tratamentos T1 $\left(0,92 \mathrm{~g} \cdot \mathrm{cm}^{-3}\right)$ e para T3 $\left(0,88 \mathrm{~g} \cdot \mathrm{cm}^{-3}\right)$. Entretanto, esses valores de densidade podem ser considerados como uma característica adequada para biocombustível sólido.

Com relação à resistência mecânica, para o ensaio de tração por compressão diametral, o tratamento que obteve maior resistência à força aplicada foi o tratamento T1 (serragem de eucalipto).

Tabela 3. Características mecânicas de briquetes de serragem de eucalipto, serragem de pinus, bagaço de cana-de-açúcar e palha de cana-de-açúcar.

Table 3. Mechanical properties of eucalyptus sawdust briquettes, pine sawdust, sugarcane bagasse and sugarcane straw.

\begin{tabular}{|c|c|c|c|}
\hline Tratamentos & Expansão longitudional(\%) & Densidade (g.cm $\left.{ }^{-3}\right)$ & Resistência (MPa) \\
\hline T1 & $9,00 \mathrm{~d}$ & $0,92 \mathrm{c}$ & $1,215 \mathrm{a}$ \\
\hline $\mathrm{T} 2$ & $7,10 \mathrm{~b}$ & $1,00 \mathrm{~b}$ & $0,697 \mathrm{c}$ \\
\hline $\mathrm{T} 3$ & $8,10 \mathrm{c}$ & $0,88 \mathrm{~d}$ & $0,270 \mathrm{~d}$ \\
\hline $\mathrm{T} 4$ & $3,85 \mathrm{a}$ & $1,11 \mathrm{a}$ & $0,975 \mathrm{~b}$ \\
\hline $\mathrm{F}$ & $* * *$ & $* * *$ & $* * *$ \\
\hline $\mathrm{CV}(\%)$ & 11,54 & 0,884 & 4,875 \\
\hline
\end{tabular}

Os resultados de expansão longitudinal de todos os tratamentos podem ser considerados baixos. Isso indica uma boa estabilidade dimensional dos briquetes. Gonçalves et al. (2013) e Chrisostomo (2011) obtiveram valores de expansão de $15,06 \%$ e 9,44\% respectivamente. Geralmente, a maior estabilidade dimensional resulta em briquetes com maior resistência mecânica. A resistência mecânica do tratamento T1 (1,215 MPa) também foi superior ao encontrado pelos mesmos autores (em média 1,00 $\mathrm{MPa})$.

De acordo com o trabalho de Oliveira et al. (1992) os resultados quantitativos da friabilidade (Figura 3) podem fornecer resultados qualitativos. Assim, os corpos de prova do tratamento T3 (bagaço de cana-de-açúcar) foram classificados como muito friável. O tratamento T2 (serragem de pinus) se enquadrou como de friabilidade média. Os tratamentos T1 (serragem de eucalipto) e T4 (palha de canade-açúcar) foram classificados como pouco friável. Quanto menor a friabilidade, mais resistente é o briquete. Assim é desejável que os briquetes apresentem os menores valores nesse ensaio $\mathrm{O}$ ensaio de friabilidade complementa o ensaio de tração por compressão diametral. Os dois ensaios caracterizam a resistência mecânica dos briquetes.

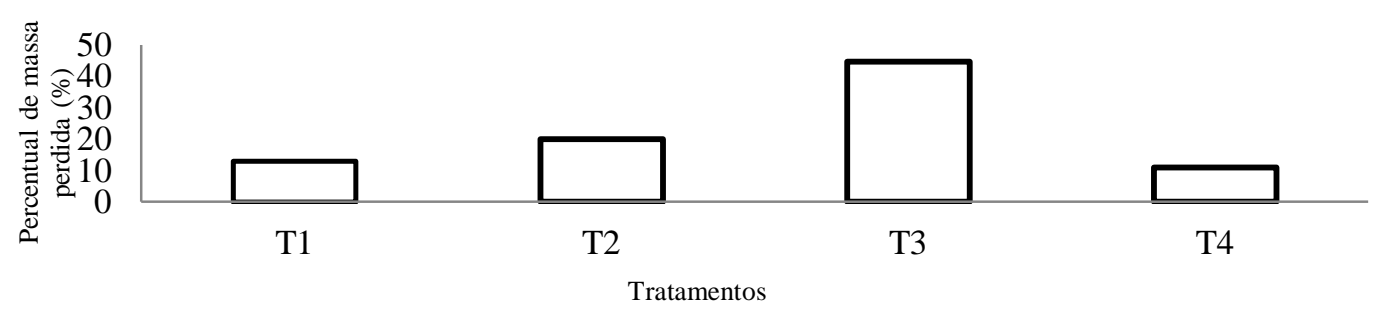

Figura 3. Teste de friabilidade dos tratamentos. Valores porcentuais da massa passante pela peneira de abertura de $4 \mathrm{~mm}$.

Figure 3. Friability test treatments. Percentages for mass passing by $4 \mathrm{~mm}$ aperture sieve.

FLORESTA, Curitiba, PR, v. 45, n. 4, p. 713 - 722 , out. / dez. 2015.

Silva, D. A. da. et al.

ISSN eletrônico 1982-4688 / ISSN impresso 0015-3826 
A altura máxima de empilhamento pode ser vista na tabela 4. Para o tratamento que apresentou a menor resistência mecânica T3 (bagaço de cana-de-açúcar), a altura máxima de empilhamento calculada foi de $20 \mathrm{~m}$. Considerando que a altura média dos galpões são inferiores a $10 \mathrm{~m}$ de altura, o empilhamento máximo seria de $10 \mathrm{~m}$ de altura. Assim, pode-se considerar que todos os tratamentos apresentam condições ótimas para empilhamento.

Tabela 4. Características de empilhamento para cada tratamento.

Table 4. Stacking characteristics for each treatment.

\begin{tabular}{lccc}
\hline Tratamentos & $\mathbf{F}_{\text {Mrup }}(\mathbf{k g f})$ & $\mathbf{N}_{\text {emp }}$ & $\mathbf{H}_{\text {emp }}(\mathbf{m})$ \\
\hline T1 & 149,90 & 2498 & 87 \\
T2 & 78,91 & 1315 & 46 \\
T3 & 35,09 & 585 & 20 \\
T4 & 100,70 & 1678 & 59 \\
\hline
\end{tabular}

A figura 4 mostra as curvas da expansão em altura dos briquetes. Todos os briquetes tendem a ter uma expansão longitudinal após o processo de briquetagem. A expansão varia dependendo do tipo de biomassa e das condições de armazenamento. Observa-se que 25h após a briquetagem os tratamentos T1, T3 e T4 atingiram a estabilidade dimensional. Os briquetes do tratamento T2 atingiram a estabilidade com 50h após a briquetagem. $\mathrm{O}$ ensaio de tração por compressão diametral foi realizados 10 dias após a briquetagem.

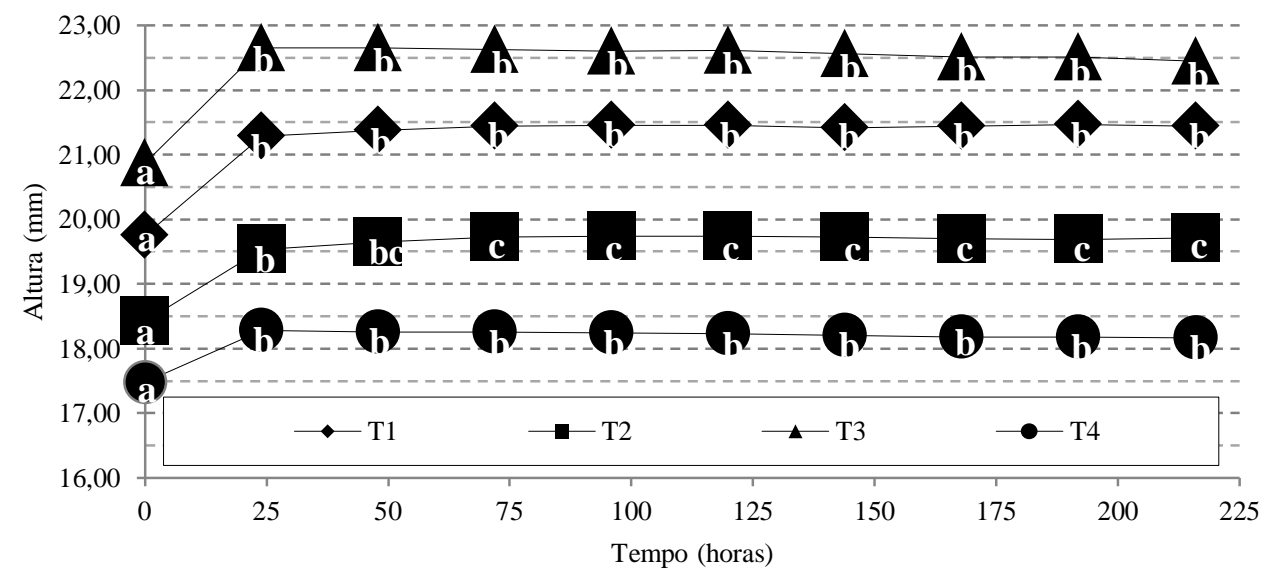

Figura 4. Curva de estabilidade dimensional dos briquetes. Dentro de cada tratamento as médias seguidas da mesma letra não diferem estatisticamente entre si pelo teste Tukey ( $\mathrm{P}>0,05)$.

Figure 4. Dimensional stability curve of the briquettes. Within each treatment means followed by the same letter do not differ significantly by the Tukey $(\mathrm{P}>0,05)$.

O ensaio de estabilidade dimensional também é importante para definir o tempo mínimo de espera para realização do ensaio de resistência mecânica. Para ser representativo, o ensaio mecânico deve ser realizados após os briquetes atingirem a estabilidade dimensional. Nos trabalhos de Chrisostomo (2011), Barros (2014) e Brasil et al. (2015) a estabilidade dimensional foi atingida após 24h da briquetagem. Os ensaios mecânicos foram realizados no mínimo $72 \mathrm{~h}$ após o processo de briquetagem. Brasil et al. (2015) acompanharam a expansão longitudinal durante 15 dias e realizaram os ensaios mecânicos 360h após a briquetagem. Após atingir a estabilidade dimensional os briquetes não sofrem alterações significativas se as condições de armazenamento forem controladas.

\section{CONCLUSÕES}

- Todos os quatro tipos de biomassa (serragem de eucalipto, serragem de pinus, bagaço de cana-deaçúcar e palha de cana-de-açúcar) mostraram-se viáveis para o processo de briquetagem.

- A granulometria do material é uma importante característica da biomassa e interfere no processo de briquetagem. 
- O ensaio mecânico de tração por compressão diametral pode auxiliar no processo de estocagem. Com os resultados dos ensaios é possível calcular a altura máxima de empilhamento dos briquetes.

\section{AGRADECIMENTOS}

Os autores agradecem ao apoio financeiro do CNPq para o desenvolvimento do projeto.

\section{REFERÊNCIAS}

ASSOCIAÇÃO BRASILEIRA DE PRODUTORES DE FLORESTAS PLANTADAS (ABRAF). Anuário Estatístico da ABRAF 2013: ano base 2012. Brasília, 2013. 148 p.

AMERICAN SOCIETY FOR TESTING AND MATERIALS. ASTM D1102-84. Standard Test Method for Ash in Wood. 2007.

ASSOCIAÇÃO BRASILEIRA DE NORMAS TÉCNICAS (ABNT). NBR 8112. Carvão vegetal: análise imediata. Rio de Janeiro, 1986.

. NBR 8633. Carvão vegetal: determinação do poder calorífico. Rio de Janeiro, 1984.

NBR 8740. Carvão Vegetal: determinação do índice de quebra e abrasão. Rio de Janeiro, 1985.

NBR 7222. Argamassa e concreto: determinação da resistência à tração por compressão diametral de corpos-de-prova cilíndricos. Rio de Janeiro: 1994.

BARROS, J. L. Caracterização de blendas e briquetes de carvões vegetal e mineral. $121 \mathrm{f}$. Dissertação (Mestrado em Ciências dos Materiais) - Universidade Federal de São Carlos, Sorocaba, 2014.

BRASIL, D. S.; MARTINS, M. P.; NAKASHIMA, G. T.; YAMAJI, F. M. Use of sugarcane bagasse and candeia waste for solid biofuel production. Floresta, Curitiba-PR, v. 45, n. 1, p. 185 - 192, 2015.

COMPANHIA NACIONAL DE ABASTECIMENTO (CONAB) Acompanhamento da safra brasileira: cana-de-açúcar. Disponível em: < http://www.conab.gov.br/OlalaCMS/uploads/arquivos/14_ 12_19_09_02_49_boletim_cana_portugues_-_3o_lev_-_2014-15.pdf $>$. Acesso em: 02 de abril de 2015

COSTA, D. R.; YAMAJI, F. M.; VENDRASCO, L.; FLORES, W. P.; CHRISOSTOMO, W. Efeito da Granulometria nas propriedades físico-mecânico de briquetes de pinus sp.. In: Congresso Internacional de Bioenergia, 5.., 2010, Curitiba. Anais do .... Curitiba: FIEP, 2010. 1 CD-ROM.

CHRISOSTOMO, W. Estudo da compactação de resíduos lignocelulósicos para utilização como combustível sólido. 56 f. Dissertação (Mestrado em Ciências dos Materiais) - Universidade Federal de São Carlos, Sorocaba, 2011.

EMPRESA DE PESQUISA ENERGÉTICA (EPE). Balanço Energético Nacional 2013: Ano Base 2012. Disponível em: < https://ben.epe.gov.br/downloads/Relatorio_Final_BEN_2013.pdf>. Acesso em: 02 de dezembro de 2014.

FERRAGUTTI, A. C.; YAMAJI, F. M.; SILVA, D. A.; QUADROS, T. M. C.; PIRES, A. A. F. Efeito da granulometria na compactação da biomassa de capim elefante. In: Encontro Brasileiro em Madeiras e em Estruturas de Madeira - EBRAMEM, 8., 2012, Vitória Anais do ... Vitória: UFES, 2012. 1 CD-ROM.

FLORES, W. P.; YAMAJI, F. M.; VENDRASCO, L.; COSTA, D. R. Redução do volume de biomassa no processo de briquetagem. Revista da Madeira. Curitiba-PR, ano 20, n. 121, p. 32 - 34, 2009.

GOLDEMBERG, J.; LUCON, O. Energia e meio ambiente no Brasil. Estudos Avançados. São PauloSP, v. 21, n. 59, p. 7 - 20, 2007.

GONÇALVES, B. F.; YAMAJI, F. M.; FERNANDEZ, B. O.; RÓZ, A.; FlORIANO, F. S. Caracterização e comparação entre diferentes granulometrias de serragem de Eucalyptus grandis para confecção de briquetes. In: Simpósio de Meio Ambiente e Tecnologia Flore, 3., 2013, Sorocaba Anais do ... Sorocaba: UFSCAR, 2013. 1 CD-ROM.

FLORESTA, Curitiba, PR, v. 45, n. 4, p. 713 - 722 , out. / dez. 2015.

Silva, D. A. da. et al.

ISSN eletrônico 1982-4688 / ISSN impresso 0015-3826

DOI: $10.5380 /$ rf.v45i4.39700 
INDÚSTRIA BRASILEIRA DE ÁRVORES (IBA). Relatório 2015. Disponível em: http://www.iba.org/pt/biblioteca-iba/publicacoes. Acesso em 31/08/2015.

KONISHI, P. A.; YAMAJI, F. M.; SILVA, D. A.; WANDERLEY, C. W. C. Influência do teor de umidade na confecção de briquetes de serragem de Pinus SP. In Congresso de Iniciação Cientifica, XIX..., 2011, São Carlos. Anais do ... São Carlos: UFSCar, 2011. 1 CD-ROM.

LUCENA, D. A.; MEDEIROS, R. D. de; FONSECA, U. T.; ASSIS, P. S. Aglomeração de moinha de carvão em alto-forno e geração de energia. Tecnologia em Metalúrgica e Materiais, São Paulo, v. 4, n. 4, p.1 - 6, 2008.

NAKASHIMA, G. T. Produção de briquetes a partir de resíduos vegetais. 18 f. Dissertação (Graduação em Engenharia Florestal). Universidade Federal de São Carlos, Sorocaba, 2013.

OLIVEIRA, J. B.; GOMES, P. A.; ALMEIDA, M. D. Estudos Preliminares de Normalização de Testes de Controle de Qualidade do Carvão Vegetal. Fundação Centro Tecnológico de Minas Gerais, Belo Horizonte. 1992.

QUIRINO, W. F. Briquetagem de Resíduos Ligno-Celulósicos. CIRCULAR TÉCNICA DO LPF, Brasília, v. 1, n. 2, p. 69 - 80, 1991.

QUIRINO, W. F. Utilização energética de resíduos vegetais. Disponível em: <http://www.mundo florestal.com.br/arquivos/aproveitamento.pdf >. Acesso em 09/10/2014.

SÃO PAULO (Estado). Lei n. 11.241, de 19 de setembro de 2002. Dispõe sobre eliminação gradativa da queima da palha da cana-de-açúcar. Diário Oficial do Estado de São Paulo, São Paulo, SP, 20 set. 2002. Disponível em: 〈http://www.al.sp.gov.br/norma/?id=217>. Acesso em: 22 jan. 2013.

VAlE, A. T. do.; BRASIL, M. A. M.; CARVALHO, C . M. de.; VEIGA, R. A. de A. Produção de energia do fuste de Eucalyptus grandis Hill Ex- Maiden e Acacia mangium Willld em diferentes níveis de adubação. Revista Cerne, v. 6, n. 1, p. 83 - 88, 2000.

YAMAJI, F. M.; VENDRASCO, L.; CHRISOSTOMO, W.; FLORES, W. P. Análise do comportamento higroscópico de briquetes. Energia na agricultura. v. 28, n. 1, p. 11 - 15, 2013. 